\title{
Quantum Beats from Nuclei Excited by Synchrotron Radiation
}

\author{
E. Gerdau, R. Rüffer, and R. Hollatz \\ II. Institut für Experimentalphysik, Universität Hamburg, D-2000 Hamburg 50, Federal Republic of Germany
}

and

J. P. Hannon

Physics Department, Rice University, Houston, Texas 77001

(Received 15 July 1986)

\begin{abstract}
We report the experimental observation of quantum beats in the coherently scattered radiation from nuclei excited by synchrotron-radiation pulses. The measurements were made with ${ }^{57} \mathrm{Fe}$ enriched yttrium-iron-garnet crystals, by reflection from two parallel crystals using the pure nuclear (002) Bragg reflection. The resulting beat pattern gives a sensitive direct measure both of the hyperfine splittings of individual iron nuclei, and of the energy shifts between different nuclear sites.

PACS numbers: $61.10 . \mathrm{Pa}, 07.85 .+\mathrm{n}, 42.10 . \mathrm{Qj}, 76.80 .+\mathrm{y}$
\end{abstract}

In a previous experiment, ${ }^{1}$ the possibility of using resonant Mössbauer filters to monochromatize synchrotron radiation to about $10^{-8} \mathrm{eV}$ in the $1-\AA \mathrm{x}$-ray region was demonstrated. This was achieved by double nuclear Bragg diffraction from ${ }^{57} \mathrm{Fe}$-yttrium-irongarnet (YIG) single-crystal films set for the electronically forbidden (002) reflection. Both the frequency spectrum and time response were measured. With an improved time resolution, we have now observed the predicted quantum beats from nuclei excited by synchrotron radiation. ${ }^{2,3}$

As pointed out by Trammel and Hannon, ${ }^{2}$ there will be quantum beats in the time spectrum for both the coherently and the incoherently scattered $\gamma$ rays and for the conversion electrons, arising from interference between the various hyperfine transitions. The essential feature is that the sharp synchrotron-radiation pulse excites the various nuclear-excited-state sublevels suddenly and coherently, which then "oscillate" at their various natural frequencies, giving beats at the difference frequencies in subsequent decays. For the incoherent processes, there is only interference between transitions to the same ground state, so that here the beat frequencies reflect the hyperfine levels of the excited state, with no information about the ground-state splittings or of the shifts between different sites. For the coherent scattering, there will be beats

$$
\Omega_{B}\left(m, n, \rho ; m^{\prime}, n^{\prime}, \boldsymbol{\rho}^{\prime}\right)=\omega_{m n}(\rho)-\omega_{m^{\prime} n^{\prime}}\left(\boldsymbol{\rho}^{\prime}\right),
$$

corresponding to the difference frequencies of all allowed nuclear transitions $\omega_{m n}$ ( $\rho$ ) from all the different nuclear sites $\rho$, from which the hyperfine splitting of both the excited and ground states may be found, as well as any energy shifts between different sites.

Our experiment measures the quantum beats of the $\gamma$ rays coherently scattered from YIG. The experimental arrangement at Hamburger Synchrotron- strahlungslabor (HASYLAB) (Deutsches ElektronenSynchrotron DESY) was the same as in the previous experiment, ${ }^{1}$ with the exception that the Ge detector was replaced by a NE102 plastic scintillator with an XP2020Q phototube. This improved the time resolution (FWHM) from 18 to $1.7 \mathrm{~ns}$. With this time resolution the fastest components expected in the YIG time spectrum can be resolved. However, the use of the plastic scintillator leads to new background contributions to the time spectrum which must be taken into account. First, because of the poor energy resolution of the detector, there is a strong increase of the prompt time peak as inelastic scattering events and higher harmonics are no longer rejected by the electronics. Second, there is a serious time-independent background noise from the photocathode. By cooling of the photocathode to $-20^{\circ} \mathrm{C}$ and proper setting of the threshold of the constant fraction discriminator, this rate was reduced to $0.1 \mathrm{~Hz}$, well below the $1-\mathrm{Hz}$ resonant signal. Finally, there is a background due to residual gas ions created in the phototube by the prompt pulse, giving a delayed contribution which starts around $240 \mathrm{~ns}$. Fortunately, this delay is greater than the quantum beat periods for YIG.

The results of our measurements are shown in Fig. 1. The solid line gives the theoretical calculation, which we now discuss.

The possibility of pure nuclear Bragg reflections in ${ }^{57} \mathrm{Fe}$-YIG has been discussed by Winkler et $a l^{4}$ The main points are as follows: YIG has a complex unit cell with $40 \mathrm{Fe}$ atoms which are distributed over $16 a$ sites (each surrounded by a distorted oxygen octahedron) and $24 d$ sites (each surrounded by a distorted oxygen tetrahedron). The $24 d$ sites are further subdivided into three equal sets by the direction of the electric-field-gradient (EFG) symmetry axis relative to the cubic crystal axes, with the $d_{1}, d_{2}$, and $d_{3}$ sites 


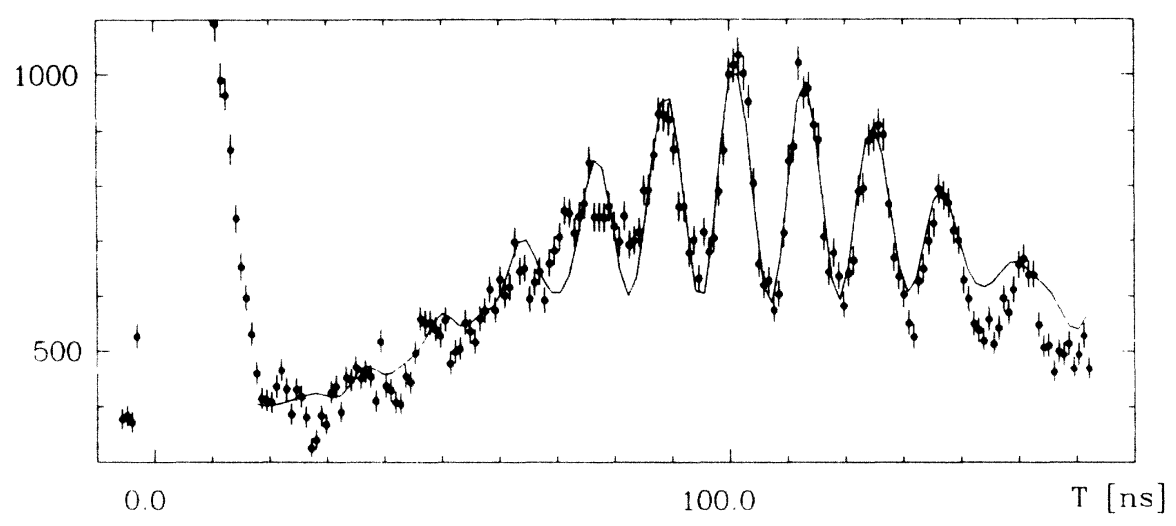

FIG. 1. Time spectrum of the coherently scattered radiation. The solid line is the calculated theoretical spectrum, plus a constant background contribution. The ratio of delayed to prompt counts is about $5 \times 10^{-3}$. The data were accumulated over three days.

having the symmetry axes parallel to the [100], [010], and [001] directions, respectively. At the $a$ sites the symmetry axis lies along one of the cube diagonals, leading to four subgroups. In addition to the fixed EFG at each site, each Fe nucleus is also acted on by the internal hyperfine field $\mathbf{B}_{\text {int }}$. The direction of $\mathbf{B}_{\text {int }}$ can be controlled with a weak applied external field $\mathbf{B}$, with $\mathbf{B}_{\text {int }}$ being parallel to the applied field at all $d$ sites, and antiparallel at the $a$ sites. At each site, the nuclear energy levels are split under the combined influence of the internal magnetic field and the EFG, with the shift being dependent on the orientation angle $\beta$ of $\mathbf{B}_{\text {int }}$ relative to the direction of the EFG symmetry axis at that site. We took $B$ along the [100] crystal axis so that $\beta=0^{\circ}$ for $d_{1}, 90^{\circ}$ for $d_{2}$ and $d_{3}$, and $54.7^{\circ}$ for all $a$ sites. The absorption spectrum is thus a superposition of three sextuplets. ${ }^{4}$

Because there are two inequivalent $d$ sites for the nuclear transitions, the electronically forbidden (002) Bragg reflection is a pure nuclear reflection. An inspection of the spatial distribution ${ }^{4}$ shows that for this reflection the $d_{1}$ and $d_{2}$ subgroups contribute with a relative phase of $180^{\circ}$. The electronic scattering from the $d_{1}$ and $d_{2}$ sites thus cancels out, leaving only the nuclear contribution. The remaining reflections from the $d_{3}, a, \mathrm{Y}$, and $\mathrm{O}$ planes are independently destructive, eliminating all other electronic and nuclear scattering.

We oriented the crystal such that the [100] crystal axis (and the applied field B) lies in the scattering plane $\left(\hat{k}_{0}, \hat{k}_{f}\right)$ of the incident and reflected beams. Because of the small Bragg angle $\left(\phi_{\mathrm{B}}=4^{\circ}\right)$ for the (002) reflection of $14.4-\mathrm{keV}$ radiation, the incident and reflected beams are nearly parallel to $B_{\text {int }}$ at the nuclear sites, and as a consequence, the right- and left-hand circular polarizations are very nearly eigenpolarizations for the scattering, with $\hat{\boldsymbol{\epsilon}}_{(+1)}$ coupling only to the
" $\Delta J_{z}$ " $=+1$ transitions $\left(J_{z}\right.$ is not precise for crossed B and EFG), and $\hat{\epsilon}_{(-1)}$ coupling only to the " $\Delta J_{z}$ " $=-1$ transitions (see Fig. 2). Since the $\hat{\boldsymbol{\epsilon}}_{( \pm 1)}$ polarizations are orthogonal, there will be separate interference in the scattered radiation between the waves from the four " $\Delta J_{z}$ " $=+1$ transitions, and between the waves from the four " $\Delta J_{z}$ " $=-1$ transitions. In the Born approximation limit, the $\pm \frac{1}{2} \hookrightarrow \pm \frac{3}{2} d_{1}$ and $d_{2}$ transitions have relative strength 1 , the $\mp \frac{1}{2} \hookrightarrow \pm \frac{1}{2}$ have strength $\frac{1}{3}$, and as already noted, the $d_{1}$ and $d_{2}$ contributions are $180^{\circ}$ out of phase. With these amplitudes and phases, the four component waves sum up to give

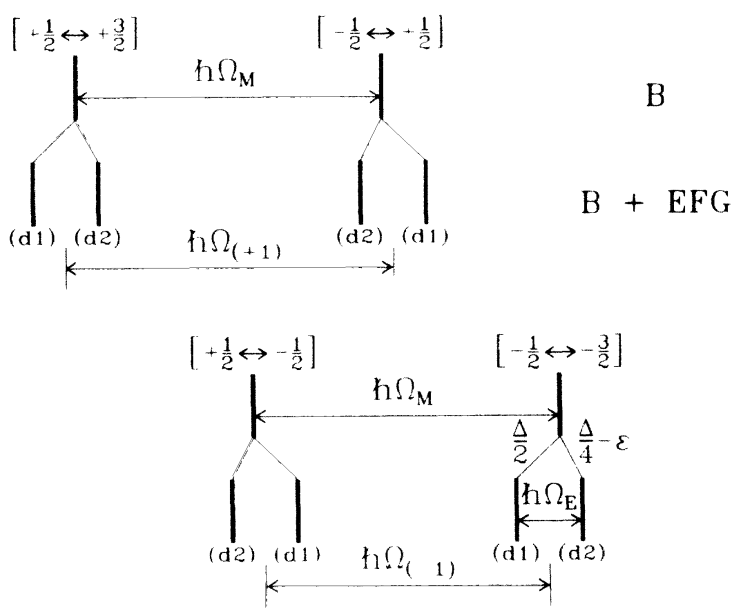

FIG. 2. Hyperfine splittings between the four " $\Delta J_{z}$ ", $=+1$ transitions and between the four " $\Delta J_{z}$ " $=-1$ transitions at the $d_{1}$ and $d_{2}$ sites with $\mathbf{B}_{\text {int }}$ along the [100] crystal axis. From Ref. 4, the room-temperature values are $\hbar \Omega_{M}=77.0 \Gamma, \quad \hbar \Omega_{E}=6.54 \Gamma, \quad \hbar \Omega_{(+1)}=79.6 \Gamma, \quad \hbar \Omega_{(-1)}$ $=74.3 \Gamma, \Delta=9.17 \Gamma$, and $\epsilon=0.34 \Gamma$ with $\Gamma=4.665 \times 10^{-9} \mathrm{eV}$. 
a simple amplitude-modulated time spectrum for each polarization. For an $m$-fold reflection, the resonant intensity for the $\hat{\boldsymbol{\epsilon}}_{( \pm 1)}$ contribution is

$$
I_{( \pm 1)}(t) \propto e^{-\Gamma t / \hbar}\left[\left(\Omega_{E} t / 2\right)^{m} j_{m-1}\left(\Omega_{E} t / 2\right)\right]^{2}\left[1+a_{m} \cos \left(\Omega_{( \pm 1)} t\right)\right] .
$$

Here $j_{m-1}$ is the spherical Bessel function of order $m-1$, and the coefficient $a_{m}$ is $a_{1}=-\frac{3}{5}$ for a single reflection, and $a_{2}=\frac{9}{41}$ for a double reflection lin this case we are dropping contributions of order $\left(\Omega_{E} /\right.$ $\left.\Omega_{M}\right)^{2}$ ]. As shown in Fig. $2, \hbar \Omega_{E}$ is the EFG splitting between adjacent $d_{1}$ and $d_{2}$ lines, and $\hbar \Omega_{( \pm 1)}$ is the splitting between the centers of the two pairs of contributing lines. Because of the effect of the EFG, this central frequency is higher for the +1 transitions than for the -1 transitions. From the symmetry of the figure, one sees that $\left[\Omega_{(+1)}+\Omega_{(-1)}\right] / 2=\Omega_{M}$, the magnetic splitting frequency, while $\Delta \Omega=\left[\Omega_{(+1)}\right.$ $\left.-\Omega_{(-1)}\right] / 2=[\Delta / 4+\epsilon] / \hbar$ is determined entirely by the EFG splitting.

The leading factor,

$$
e^{-\Gamma t / \hbar}\left[\left(\Omega_{E} t / 2\right)^{m} j_{m-1}\left(\Omega_{E} t / 2\right)\right]^{2},
$$

gives a slowly developing overall modulation which arises from the interference between the adjacent
EFG-split lines. This factor is initially zero (because of the $180^{\circ}$ phase shift between the lines), and builds up to a peak response at approximately $t_{\max }(\mathrm{m})$ $\approx m \pi / \Omega_{E}$, followed by damped oscillations of approximate period $\tau_{E} \approx 2 \pi / \Omega_{E}$ (the response is simply periodic only for $m=1$ ). For a single reflection, the time delay for peak response, $\approx \pi / \Omega_{E}=68 \mathrm{~ns},{ }^{5}$ is just the time required for two oscillators to first get in phase, when started $180^{\circ}$ out of phase with frequencies $\omega$ and $\omega+\Omega_{E}$. For $m>1$, there is an additional multiple-reflection delay for peak response as we have discussed before. ${ }^{3}$

The factor $1+a_{m} \cos \left(\Omega_{( \pm 1)} t\right)$ superimposes a fast ringing on the slowly developing envelope. Because of the slight shift between the \pm 1 frequencies, the superposition of the \pm 1 components will give a further modulation of the fast beats. For incident linearly polarized synchrotron radiation, the time response is $I(t)=\frac{1}{2}\left[I_{(+1)}+I_{(-1)}\right]$, which is given by

$$
I(t) \propto e^{-\Gamma t / \hbar}\left[\left(\Omega_{E} t / 2\right)^{m} j_{m-1}\left(\Omega_{E} t / 2\right)\right]^{2}\left[1+a_{m} \cos (\Delta \Omega t) \cos \left(\Omega_{M} t\right)\right] .
$$

Thus, if the polarization of the scattered radiation is not detected, the fast ringing is precisely equal to the magnetic hyperfine splitting $\Omega_{M}$, giving a fast beat period of $\tau_{M}=11.5 \mathrm{~ns}^{5}$ There is an additional slow amplitude modulation at the difference frequency $\Delta \Omega$ which is determined entirely by the EFG splitting.

Although the Born approximation gives one a good understanding of the qualitative features of the time response, for an accurate fit to the data one must use the full dynamical theory. ${ }^{3,6-8}$ In the notation of Ref. 3 , following excitation by a synchrotron pulse of polar- ization $\hat{\boldsymbol{\epsilon}}_{0}$ incident at $t=0$, the time-dependent coherently scattered photon flux is

$$
I(t)=\Delta_{y} \int_{\Delta_{x}} d \phi\left[2 \pi \hbar I_{0 p}(\phi)\right]\left|\tilde{R}(t, \phi) \hat{\epsilon}_{0}\right|^{2} .
$$

$\Delta_{x}$ is the vertical divergence of the synchrotron beam and $\tilde{R}(t, \phi)$ gives the amplitude response of the system to a $\delta$-function pulse,

$$
\tilde{R}(t, \phi)=(2 \pi)^{-1} \int_{-\infty}^{+\infty} d \omega[\tilde{R}(\omega, \phi)]^{m} e^{-i \omega t}
$$

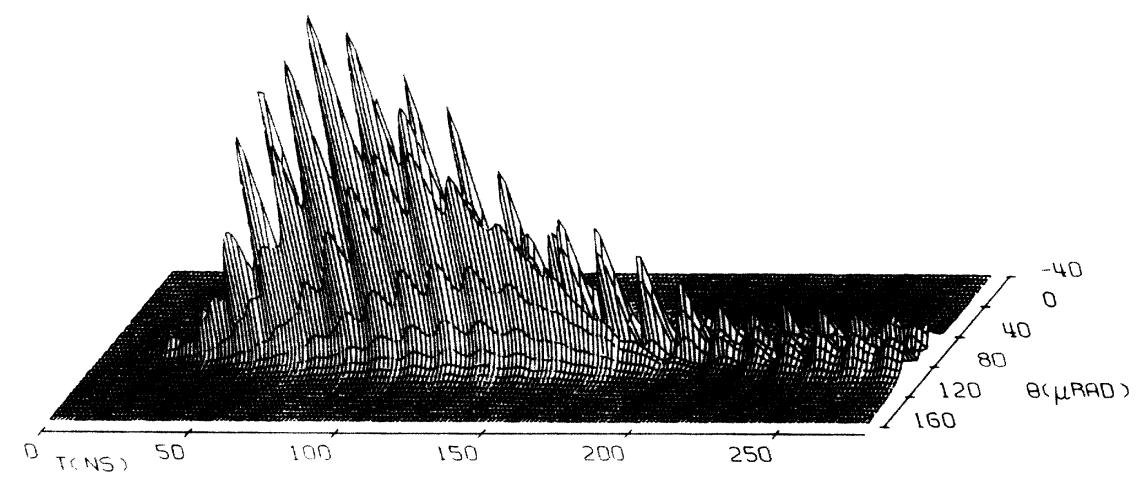

FIG. 3. 3D plot of the time response $\left|\tilde{R}(t, \phi) \hat{\boldsymbol{\epsilon}}_{\sigma}\right|^{2}$ following a double (002) Bragg reflection of incident radiation of polarization $\sigma$ from YIG. $\mathbf{B}_{\text {int }}$ and the scattering plane $\left(\hat{k}_{0}, \hat{k}_{f}\right)$ are parallel to the $[100]$ crystal axis. 
for an $m$-fold reflection from parallel crystal films. $\tilde{R}(\omega, \phi)$ is the $2 \times 2$ reflectivity matrix for the coherent elastic reflection from a single crystal of planewave radiation of frequency $\omega$ at incidence angle $\phi$. We have calculated $\tilde{R}(\omega, \phi)$ using the full determinant solution ${ }^{6}$ to account for polarization mixing, and using the diagonalization procedure given in Refs. 4 and 6 to get the proper scattering amplitudes for nuclei under the influence of a crossed EFG and $\mathbf{B}_{\text {int }}$. Figure 3 gives a $3 \mathrm{D}$ plot of the resulting time response, $\left|\tilde{R}(t, \phi) \hat{\boldsymbol{\epsilon}}_{\sigma}\right|^{2}$, for a double reflection from YIG of incident radiation of polarization $\sigma$. This must be integrated over the beam divergence and any mosaic spread of the crystals. More generally, one must also allow for angular deviations between crystals in a multiple reflection.

Although the dynamical calculation is considerably more involved than the Born approximation, the theoretical fit still depends very sensitively on the hyperfine parameters. For our particular YIG reflection, although the dependence is more complex, it is still true that the delay to peak response, the period of the slow oscillations, and the positions of destructive cancellation in the fast beat period are all determined by the EFG splitting, while the fast beat pattern is determined by the magnetic splitting. ${ }^{8}$

The solid line in Fig. 1 gives our calculation for the time response. Here we have taken an incident Gaussian spread with $\sigma=12 \mu \mathrm{rad}$ centered at peak Bragg reflectivity. Optimum simulation of the spectrum is obtained with $\hbar \Omega_{E}=6.5 \Gamma$ and $\hbar \Omega_{M}=73.9 \Gamma$. The latter value represents a $4 \%$ shift from the data of Ref. 4 . This is explained in part by a higher operating temperature, and by a correction of $2 \%$ to the previous determinations. ${ }^{9}$

In summary, we have observed hyperfine quantum beats from nuclei excited by synchrotron-radiation pulses, and have demonstrated that it is possible to determine magnetic and EFG hyperfine splittings. The time measurements have the potential for giving more accurate determinations of hyperfine splittings than absorption measurements because the energy separations are directly determined by the beat frequencies, and because the measurements are on a direct time scale rather than on a calibrated velocity scale. We will carry out measurements with new orientations of $\mathbf{B}_{\text {int }}$ in a temperature-stabilized environment. For $\mathbf{B}_{\perp}\left(\hat{k}_{0}, \hat{k}_{f}\right)$, the beats will exhibit the splitting between the +1 and -1 resonances, in addition to their separate splittings. We are also attempting to use a single reflection in order to improve the signal and to simplify the data analysis.

We acknowledge the continuous and helpful collaboration of Professor W. Tolksdorf and Dr. C. P. Klages of the Philips Forschungslaboratorium in Hamburg who produced the enriched YIG crystals. The financial support of the Bundesministerium für Forschung und Technologie through Project No. 05269GU is gratefully acknowledged. Partial support was also provided by the Welch Foundation.

1E. Gerdau, R. Rüffer, H. Winkler, W. Tolksdorf, C. P. Klages, and J. P. Hannon, Phys. Rev. Lett. 54, 835 (1985).

${ }^{2}$ G. T. Trammell and J. P. Hannon, Phys. Rev. B 18, 165 (1978), and 19, 3835 (1979).

3J. P. Hannon, G. T. Trammell, M. Mueller, E. Gerdau, R. Rüffer, and H. Winkler, Phys. Rev. B 32, 6374 (1985).

${ }^{4}$ H. Winkler, R. Eisberg, E. Alp, R. Rüffer, E. Gerdau, S. Lauer, A. X. Trautwein, M. Grodzicki, and A. Vera, Z. Phys. B 49, 331 (1983).

${ }^{5}$ All estimates given use the room-temperature data of YIG from Ref. 4.

6J. P. Hannon and G. T. Trammell, Phys. Rev. 186, 306 (1969).

${ }^{7} Y$ u. M. Kagan, A. M. Afanas'ev, and V. G. Kohn, J. Phys. C 12, 615 (1979).

${ }^{8} \mathrm{~A}$ more detailed discussion will be published elsewhere.

${ }^{9}$ This is due to a change of the adopted value of $g_{0} / g_{1}$ in the fitting program for the YIG spectra. 


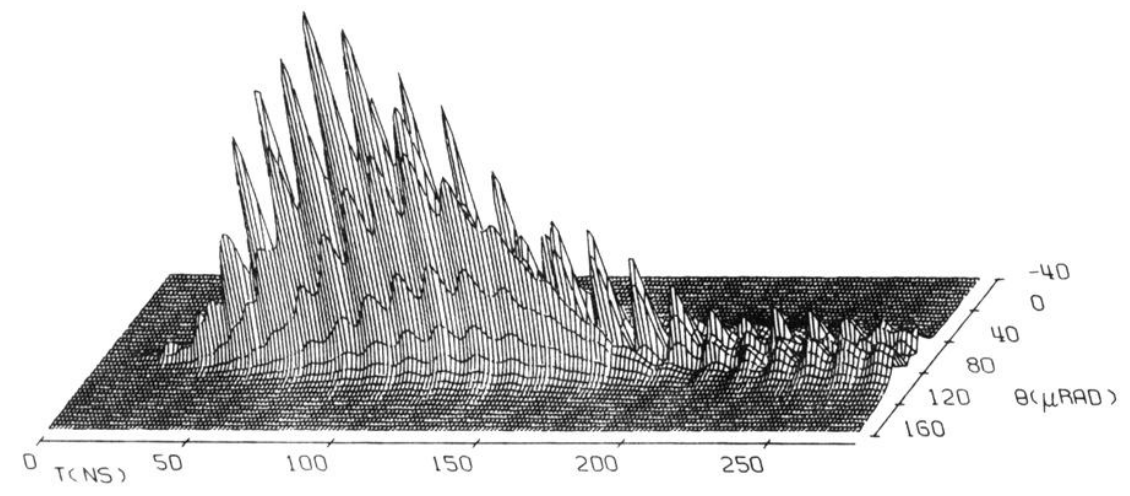

FIG. 3. 3D plot of the time response $\left|\tilde{R}(t, \phi) \hat{\boldsymbol{\epsilon}}_{\sigma}\right|^{2}$ following a double (002) Bragg reflection of incident radiation of polarization $\sigma$ from YIG. $\mathbf{B}_{\text {int }}$ and the scattering plane $\left(\hat{k}_{0}, \hat{k}_{f}\right)$ are parallel to the [100] crystal axis. 